\title{
Errata: Confocal reflectance and two-photon microscopy studies of a songbird skull for preparation of transcranial imaging
}

\author{
Darine Abi-Haidar \\ Thomas Olivier \\ University of Lyon \\ and \\ University of Saint-Etienne \\ CNRS UMR5516 \\ Laboratoire Hubert Curien \\ 18 rue Pr. Benoit Lauras \\ Saint-Etienne 42000 \\ France \\ E-mail: thomas.olivier@univ-st-etienne.fr \\ [DOI: 10.1117/1.3223351]
}

This article [J. Biomed. Opt. 14, 034038 (2009)] was originally published online on 29 June 2009 with an error in the author list. The second author should have been Thomas Olivier instead of Thomas Oliver. All online versions of the article were corrected on 1 July 2009 and the article appears correctly in print. 